\title{
Downregulation of ZNF365 by methylation predicts poor prognosis in patients with colorectal cancer by decreasing phospho-p53 (Ser 15) expression
}

\author{
CHAN WANG ${ }^{1 *}$, SHUIPING LIU ${ }^{2 *}$, YEYE KUANG ${ }^{1}$, XIAOTONG HU ${ }^{1,3}$ and XIAO FANG ${ }^{4}$ \\ ${ }^{1}$ Biomedical Research Center and Key Laboratory of Biotherapy of Zhejiang Province, Sir Run Run Shaw Hospital, \\ Zhejiang University, Hangzhou, Zhejiang 310016; ${ }^{2}$ Department of Cancer Pharmacology and Key Laboratory of Elemene \\ Class Anti-Cancer Chinese Medicine of Zhejiang Province, Holistic Integrative Pharmacy Institutes, College of Medicine, \\ Hangzhou Normal University, Hangzhou, Zhejiang 310012; Departments of ${ }^{3}$ Pathology \\ and ${ }^{4}$ Anesthesiology and Key Laboratory of Biotherapy of Zhejiang Province, Sir Run Run \\ Shaw Hospital, Zhejiang University, Hangzhou, Zhejiang 310016, P.R. China
}

Received August 8, 2019; Accepted June 26, 2020

DOI: $10.3892 / \mathrm{ol} .2020 .11946$

\begin{abstract}
ZNF365 is a transcription factor that plays important roles in different types of cancer, such as colorectal cancer, breast cancer and hepatocellular carcinoma. ZNF365 can promote stalled replication fork recovery to prevent genomic instability, which is a notable feature of sporadic and hereditary types of cancers. However, the function of ZNF365 in the tumor progression of colorectal cancer $(\mathrm{CRC})$ remains unclear. Thus, immunohistochemical staining was used to investigate the association between ZNF365 expression and the clinicopathological characteristics of patients with colorectal cancer. The results demonstrated that ZNF365 protein was strongly expressed in the nucleus and cytoplasm of normal colorectal mucosa. Furthermore ZNF365, which is methylated and downregulated in most cancer cell lines and tissues, was significantly associated with lymph node metastasis $(\mathrm{P}=0.015)$, depth of invasion $(\mathrm{P}=0.031)$ and histopathological grading $(\mathrm{P}=0.042)$. A positive correlation was observed between ZNF365 expression and phosphorylated (P)-p53 (Ser15) protein expression
\end{abstract}

Correspondence to: Dr Xiao Fang, Department of Anesthesiology and Key Laboratory of Biotherapy of Zhejiang Province, Sir Run Run Shaw Hospital, Zhejiang University, 3 Qingchun East Road, Hangzhou, Zhejiang 310016, P.R. China

E-mail: 3195046@zju.edu.cn

Dr Xiaotong Hu, Biomedical Research Center and Key Laboratory of Biotherapy of Zhejiang Province, Sir Run Run Shaw Hospital, Zhejiang University, 3 Qingchun East Road, Hangzhou, Zhejiang 310016, P.R. China

E-mail: hxt_hangzhou@zju.edu.cn

*Contributed equally

Key words: ZNF365, colorectal cancer, immunohistochemistry, DNA methylation $(\mathrm{r}=0.18 ; \mathrm{P}=0.038)$. Survival analysis indicated that patients with high ZNF365 expression had a higher survival rate than those with low $\mathrm{ZNF} 365$ expression $(\mathrm{P}=0.009)$, suggesting that ZNF365 may be an independent prognostic factor for survival in colorectal cancer $(\mathrm{P}=0.046)$. Taken together, the results of the present study demonstrated that ZNF365 was frequently inactivated by promoter methylation and independently predicted poor prognosis in patients with colorectal cancer by downregulating P-p53 (Ser15) expression.

\section{Introduction}

Colorectal cancer (CRC) is the most common gastrointestinal malignancy, as well as the third most lethal and fourth most diagnosed type of cancer in the world, despite recent advancements in the treatment of CRC (1). CRC is caused by genetic and epigenetic alterations, including histone and chromatin structural modification, DNA methylation and microRNA (miRNA) aberrations (2). DNA methylation usually leads to the hypermethylation of gene promoters and the inactivation of tumor suppressor genes (TSGs), which plays an important role in the initiation, development and recurrence of CRC (3). Thus, identification of TSGs that undergo CpG island hypermethylation and assessment of their roles and associated molecular mechanisms in tumor progression will help to develop more effective diagnosis and individualized therapeutic strategies for patients with CRC $(3,4)$.

The zinc finger proteins (ZNFs) are classified into eight-fold groups according to the secondary structure around the zinc-binding site and main chain conformation (5). ZNFs have several functions, including apoptosis regulation, transcriptional activation, protein folding and integration, RNA packaging, DNA recognition and lipid binding (6). ZNFs have been reported to play important roles in different types of human cancer. Some ZNFs act as oncogenes, for example, ZNF306 expression is upregulated in CRC, whereas low ZNF306 expression suppresses tumor development (7). Furthermore, ZNF307 inhibits the activity of tumor suppressor 
genes, p21 and p53 by increasing the transcription of EP300 and MDM2 (8). Conversely, some ZNFs act as tumor suppressors, for example, ZNF23 inhibits the proliferation of SK-OV-3 cells by enhancing p27/kip-1 expression (9). Furthermore, ZNF668 is considered a tumor suppressor in breast cancer, which stabilizes 553 by preventing MDM2-mediated ubiquitination and degradation of p53 (10).

ZNF365 contains the N-terminus-C2H2 zinc finger motif, and was first discovered in 1998 from the human brain cDNA library $(11,12)$. It has been reported that polymorphisms in the ZNF365 gene or its locus is associated with different types of disease. For example, polymorphisms in the Ala62Throf ZNF365 gene are associated with susceptibility to uric acid nephrolithiasis $(12,13)$. Furthermore, variants of ZNF365 are associated with Crohn's disease (14). A total of five single nucleotide polymorphisms (SNPs) in ADO-ZNF365-EGR2 have been demonstrated to be associated with Vogt-Koyanagi-Harada (VKH) syndrome in patients with VKH, in Thailand (15). It has been reported that genetic variations in ZNF365 affected the risk of breast cancer by influencing the proportion of dense tissue in the breast (16). Additionally, variants at the ZNF365 loci are associated with estrogen receptor subtypes of breast cancer risk in BRCA1 and BRCA2 mutation carriers (17).

Although ZNF365 is known to play important roles in different types of human cancer, its function in CRC remains unknown. Thus, the present study aimed to investigate the association between ZNF365 expression and tumor progression of $\mathrm{CRC}$, and determine its underlying molecular mechanism.

\section{Materials and methods}

Cell lines and culture conditions. The CRC cell lines (Colo320, SW620, SW480, HCT116, SW48, LOVO, HCT8, DLD1, HT29 and RKO) were purchased from The Cell Bank of Type Culture Collection of the Chinese Academy of Sciences. Cells were maintained in DMEM (Gibco; Thermo Fisher Scientific, Inc.) or RPMI-1640 (Gibco; Thermo Fisher Scientific, Inc.) medium supplemented with $10 \%$ fetal bovine serum (cat. no. VS500T; Ausbian), in a humidified $5 \% \mathrm{CO}_{2}$ incubator at $37^{\circ} \mathrm{C}$.

Tissue specimens. For immunohistochemistry (IHC) analysis, 120 patients with CRC who underwent surgery at the Sir Run Run Shaw Hospital between February 2004 and June 2006 were recruited in the present study, and 10 normal colonic mucosa biopsy samples were selected as the normal controls (4 males and 6 females; mean age, 51.12 years; age range, 32-65 years). The present study was approved by the Ethics Committee of Sir Run Run Shaw Hospital (Hangzhou, China) and all patients provided written informed consent prior to the study start (approval no. 2019ZNF365-1). Patients who had received preoperative chemotherapy, radiotherapy or immunotherapy prior to surgery were excluded. A total of 79 men and 41 women, with a mean age of 63.6 years (age range, 28-89 years) were included in the present study.

Following surgical resection, the tissue samples were fixed at room temperature in $10 \%$ formalin for $24 \mathrm{~h}$, embedded in paraffin and sectioned into 4-um-thick slices. The intensity of ZNF365 immunostaining was scored by two experienced pathologists from Sir Run Run Shaw Hospital (Hangzhou,
China) who were unaware of the clinicopathological outcomes of the patients, using the World Health Organization classification guidelines (18). A typical section for each case was selected for IHC analysis.

Differentiation status was divided into three subtypes: i) Well differentiated, including papillary adenocarcinoma and high differentiated tubular adenocarcinoma; ii) moderately differentiated, including highly to moderately differentiated tubular adenocarcinoma and iii) poorly differentiated, including poorly differentiated adenocarcinoma, signet-ring cell carcinoma, mucinous adenocarcinoma and undifferentiated carcinoma. According to these criteria, there were 82 well differentiated, 25 well/moderately differentiated and 13 poorly differentiated types of cancer of the 120 cases. Lymph node metastasis and depth of invasion were graded based on the 7th edition of the International Union Against Cancer tumor-node-metastasis (TNM) system (19). All patients were followed up via telephone for 36 months.

ZNF365 methylation was assessed in normal colorectal (NC) tissues and paired CRC tissues, which were obtained from 30 patients in July 2009. The patients included 11 males and 19 females with a mean age of 56.2 years (age range, 41-68 years). The corresponding $\mathrm{NC}$ tissues were removed from the margin of the resection with a distance $>10 \mathrm{~cm}$ away from the tumor.

Semi-quantitative reverse transcription (RT)-PCR. Semi-quantitative RT-PCR was performed as previously described (20). Total RNA was extracted from fresh cells (Colo320, SW620, SW480, HCT116, SW48, LOVO, HCT8, DLD1, HT29 and RKO) using TRIzol ${ }^{\circledR}$ reagent (Invitrogen; Thermo Fisher Scientific, Inc.). Subsequently, RNA was converted to cDNA using RevertAid First Strand cDNA Synthesis kit (cat. no. K1622; Thermo Fisher Scientific, Inc.). GAPDH mRNA was used as a control. The primers used in the present study are listed in Table SI.

Methylation analysis of ZNF365. To determine the molecular mechanism underlying aberrant ZNF365 expression in colorectal cancer, the association between ZNF365 mRNA expression and DNA methylation was assessed using the cBioPortal online database (www.cbioportal.org).

GeneMANIA analysis. GeneMANIA is a commonly used website for constructing protein-protein interaction networks and predicting the function of favorite genes (21). The GeneMANIA database (http://genemania.org/) was used to assess the association between ZNF365 and p53 expression levels.

5-Aza-2'-deoxycytidine and trichostatin A treatment. RKO cells that do not express ZNF365 were seeded into a $10-\mathrm{cm}$ dish at a density of $1 \times 10^{6}$ cells $/ \mathrm{ml}$ and cultured overnight in a humidified incubator at $37^{\circ} \mathrm{C}$ with $5 \% \mathrm{CO}_{2}$. Cells were subsequently treated with demethylating agent 5-aza (Sigma-Aldrich; Merck KGaA) at a final concentration of $10 \mathrm{mM}$ for 3 days and further treated with the histone deacetylase inhibitor TSA (Sigma-Aldrich; Merck KGaA) at a final concentration of $300 \mathrm{nmol} / \mathrm{l}$ for an additional $24 \mathrm{~h}$ at $37^{\circ} \mathrm{C}$. Cells were collected for DNA and RNA extraction. 

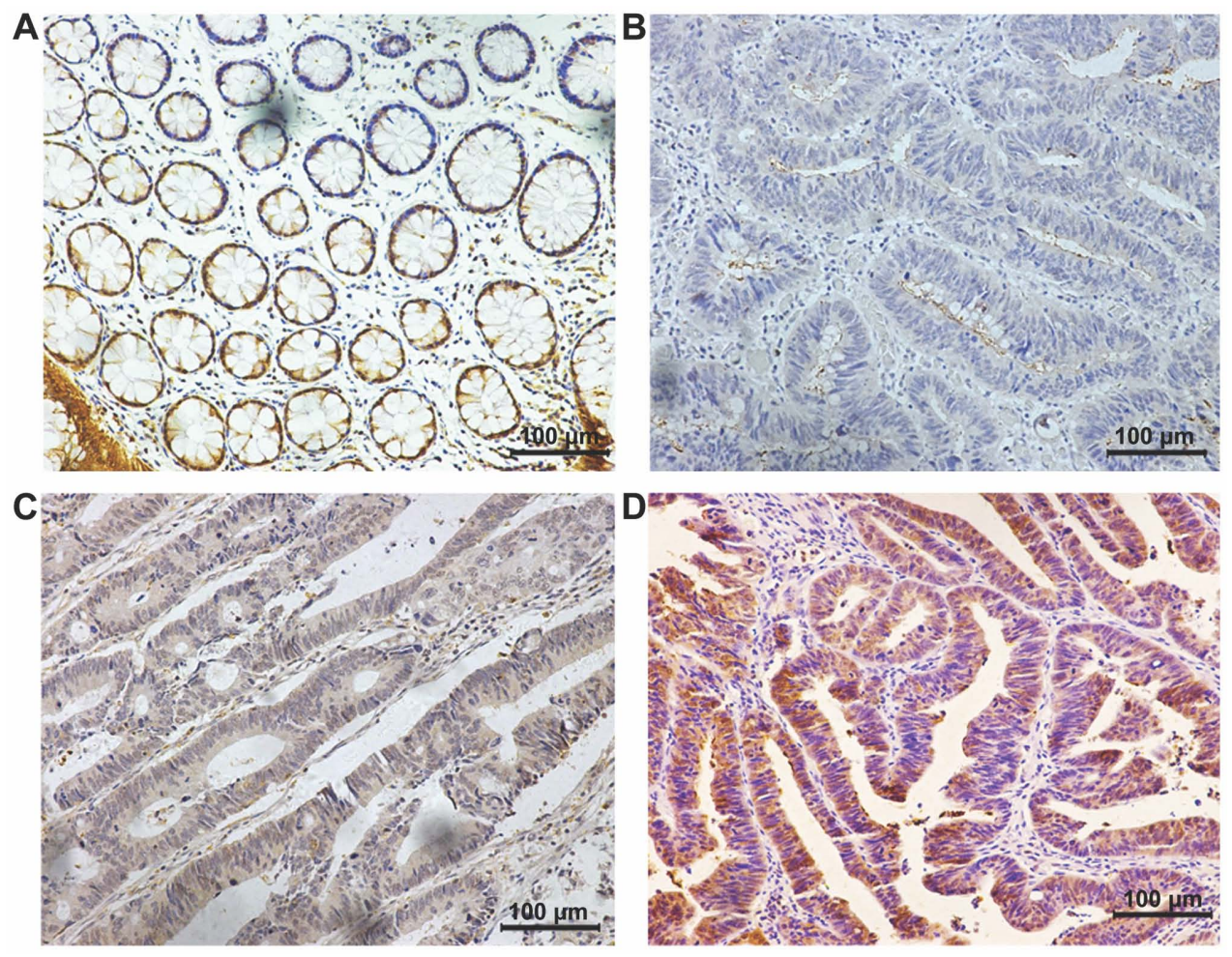

Figure 1. Representative images of immunohistochemical staining of ZNF365 in colorectal cancer tissues and normal colorectal mucosa. (A) ZNF365 expression in normal colorectal mucosa. (B) ZNF365 weak staining, (C) ZNF365 moderate staining and (D) ZNF365 strong staining in colorectal cancer tissues (magnification, x200).

Bisulfite treatment and promoter methylation analysis. Methylation-specific PCR (MSP), bisulfite modification of DNA and bisulfite genomic sequencing (BGS) were performed as previously described (22). The primer sequences used for MSP and BGS are listed in Table SI.

IHC staining. IHC staining was performed using the ChemMate $^{\mathrm{TM}}$ EnVision $^{\mathrm{TM}}$ detection kit (Dako; Agilent Technologies, Inc.) according to the manufacturer's instructions. Briefly, the CRC sections and the normal colonic mucosa biopsy samples were dewaxed and hydrated with 100\% dimethylbenzene (cat. no. 1330-20-7; Shanghai Macklin Biochemical Co., Ltd.), and rehydrated in a descending ethanol series. Tissue sections were washed with deionized water and phosphate buffered saline (PBS). The antigen retrieval process was performed with $0.01 \mathrm{M}$ citrate buffer $(\mathrm{pH} 6.0$; cat. no. C1013; Beijing Solarbio Science \& Technology Co., Ltd.). After cooling to room temperature, the tissue sections were blocked with $3 \%$ hydrogen peroxidase-methanol solution for 30 min to inhibit endogenous peroxidase activity, and subsequently incubated in preimmunized goat serum (cat. no. C0265; Beyotime Institute of Biotechnology) for $30 \mathrm{~min}$, both at room temperature. Tissue sections were incubated with rabbit polyclonal IgG primary antibody directed against ZNF365 (1:50; cat. no. HPA052446; Atlas Antibodies) overnight at $4^{\circ} \mathrm{C}$. After warming to room temperature, the tissue sections were washed five times with PBS and subsequently incubated with ChemMateEnVision/HRP, Rabbit/Mouse reagent (Dako; Agilent Technologies, Inc.) at room temperature for $30 \mathrm{~min}$. The sections were stained with ChemMate DAB+chromogen (Dako; Agilent Technologies, Inc.) and counterstained with hematoxylin at room temperature for 1 min each. Tissue sections were dehydrated in an ascending ethanol series and dimethylbenzene, and were observed using a light microscope (magnification, $\mathrm{x} 200$ ).

Evaluation of staining. A total of two independent pathologists from Sir Run Run Shaw Hospital (Hangzhou, China) blindly assessed the slides three times to determine the percentage of positive cells, staining intensity and subcellular localization. ZNF365 expression was scored using the World Health Organization classification system. The percentage of positive cells was scored as follows: $0,0-10 ; 1,11-25 ; 2,26-50$; and $3,51-100 \%$. The intensity of staining was scored as follows: 0 , negative; 1 , weak; 2 , moderate and 3 , strong. The final immunoreactivity score (IRS) was equal to the sum of both scores, which ranged from 0-9. The specimens were divided into two groups according to the IRS value, high expression group (IRS 6-9) and low expression group (IRS 0-5) in order to assess the association between ZNF365 expression and clinicopathological characteristics.

Statistical analysis. Statistical analysis was performed using SPSS 22.0 software (IBM Corp.). The Kaplan-Meier method was used to assess the survival curve and statistical differences were determined using the log-rank test. Pearson's $\chi^{2}$ test and Fisher's exact test were used to assess the association between ZNF365 expression with clinicopathological characteristics. Cox's proportional hazards regression model was used to perform univariate (depth of invasion, tumor location, distant metastasis, sex, age, differentiation, lymph node metastasis, TNM stage and ZNF365 expression) and 
Table I. Association between ZNF365 expression and clinicopathological characteristics of patients with colorectal cancer $(\mathrm{n}=120)$.

\begin{tabular}{|c|c|c|c|c|c|}
\hline \multirow[b]{2}{*}{ Characteristic } & \multirow[b]{2}{*}{ Number of patients, n (\%) } & \multicolumn{2}{|c|}{ ZNF365 expression } & \multirow[b]{2}{*}{$\chi^{2}$} & \multirow[b]{2}{*}{ P-value } \\
\hline & & Low, n (\%) & High, n (\%) & & \\
\hline Total & 120 & $54(45.000)$ & $66(55.000)$ & & \\
\hline \multicolumn{6}{|l|}{ Sex } \\
\hline Male & $79(65.833)$ & $33(41.772)$ & $46(58.228)$ & 0.973 & 0.324 \\
\hline Female & $41(34.167)$ & $21(51.220)$ & $20(48.780)$ & & \\
\hline \multicolumn{6}{|l|}{ Age, years } \\
\hline$\geq 63$ & $62(51.667)$ & $28(45.162)$ & $34(54.838)$ & 0.001 & 0.971 \\
\hline$<63$ & $58(48.333)$ & $26(44.828)$ & $32(55.172)$ & & \\
\hline \multicolumn{6}{|l|}{ Tumor location } \\
\hline Rectum & 77 (64.167) & $32(41.558)$ & $45(58.442)$ & 1.028 & 0.311 \\
\hline Colon & $43(35.833)$ & $22(51.163)$ & $21(48.837)$ & & \\
\hline \multicolumn{6}{|c|}{ Histopathological grading } \\
\hline G1 (Well) & $82(68.333)$ & $35(42.683)$ & $47(57.317)$ & 6.349 & $0.042^{\mathrm{a}}$ \\
\hline G2 (Moderate) & $25(20.833)$ & $16(64.000)$ & $9(36.000)$ & & \\
\hline G3 (Poor) & $13(10.833)$ & $3(23.077)$ & $10(76.923)$ & & \\
\hline \multicolumn{6}{|l|}{ Depth of invasion } \\
\hline $\mathrm{pT} 1 / \mathrm{T} 2$ & $34(28.333)$ & $10(29.412)$ & $24(70.588)$ & 4.658 & $0.031^{\mathrm{a}}$ \\
\hline $\mathrm{pT} 3 / \mathrm{T} 4$ & $86(71.667)$ & $44(51.163)$ & $42(48.837)$ & & \\
\hline \multicolumn{6}{|c|}{ Lymph node status } \\
\hline N0 & $68(56.667)$ & $24(35.294)$ & $44(64.706)$ & 5.973 & $0.015^{\mathrm{a}}$ \\
\hline $\mathrm{N} 1 / 2$ & $52(43.333)$ & $30(57.692)$ & $22(42.308)$ & & \\
\hline \multicolumn{6}{|l|}{ Distant metastasis } \\
\hline M0 & $101(84.167)$ & $43(42.574)$ & $58(57.426)$ & 1.517 & 0.218 \\
\hline M1 & $19(15.833)$ & $11(57.895)$ & $8(42.105)$ & & \\
\hline \multicolumn{6}{|l|}{ TNM stage } \\
\hline $\mathrm{I} / \mathrm{II}$ & $63(52.500)$ & $24(38.095)$ & $39(61.905)$ & 2.555 & 0.110 \\
\hline III/IV & $57(47.500)$ & $30(52.632)$ & $27(47.368)$ & & \\
\hline
\end{tabular}

${ }^{\mathrm{a}} \mathrm{P}<0.05 . \mathrm{pT}$, pathological tumor stage; TNM, tumor-node-metastasis.

multivariate analyses. Relative risk of mortality is presented as adjusted hazard ratios (HRs) and corresponding $95 \%$ confidence intervals $(\mathrm{CI}) . \mathrm{P}<0.05$ was considered to indicate a statistically significant difference.

\section{Results}

ZNF365 expression in CRC tissues. ZNF365 expression was assessed in 120 cases of CRC and 10 normal colonic mucosa biopsy samples were used as the normal controls. Representative immunostaining images of ZNF365 in CRC tissues are presented in Fig. 1. The results demonstrated that ZNF365 was expressed in the cytoplasm and nucleus of cancer tissues. Patients were divided into two groups according to the IRS value, high expression group (IRS 6-9) and low expression group (IRS 0-5). A total of 54 cases of CRC tissues (45\%) were classified into the low ZNF365 expression group, while the remaining 66 cases $(55 \%)$ were classified into the high ZNF365 expression group (Table I).
Association between ZNF365 expression and clinicopathological characteristics of patients with $C R C$. The association between ZNF365 expression and clinicopathological characteristics of patients with CRC are presented in Table I. The results demonstrated that ZNF365 was significantly associated with lymph node metastasis $(\mathrm{P}=0.015)$, depth of invasion $(\mathrm{P}=0.031)$ and histopathological grading $(\mathrm{P}=0.042)$. However, there were no significant associations between ZNF365 expression and age $(\mathrm{P}=0.971)$, sex $(\mathrm{P}=0.324)$, distant metastasis $(\mathrm{P}=0.218)$, tumor location $(\mathrm{P}=0.311)$ or TNM stages $(\mathrm{P}=0.11)$.

Downregulation of ZNF365 is associated with poor survival of patients with CRC. All patients were followed up for overall survival following surgery, to further determine the role ZNF365 plays in the progression of CRC. The prognosis of patients with high and low ZNF365 expression was assessed (Fig. 2). Kaplan-Meier survival analysis demonstrated that the 3-year survival rate was higher in patients with high ZNF365 


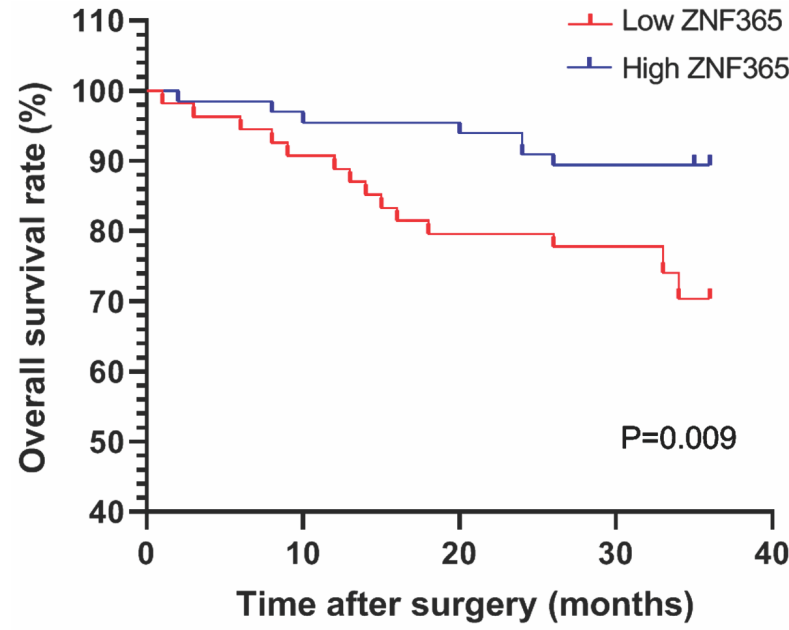

Figure 2. Kaplan-Meier survival curve of patients with colorectal cancer according to ZNF365 expression. The 3-year overall survival rate of patients with low ZNF365 expression was significantly lower than that of patients with high ZNF365 expression.

expression than those with low ZNF365 expression (Fig. 2; $\mathrm{P}=0.009)$. Univariate analysis demonstrated that in addition to ZNF365 expression ( $\mathrm{P}=0.013)$, lymph node metastasis $(\mathrm{P}<0.001)$, tumor histopathological grading $(\mathrm{P}=0.011)$, distant metastasis $(\mathrm{P}<0.001)$, depth of invasion $(\mathrm{P}=0.044)$ and TNM stages $(\mathrm{P}<0.001)$ were also significantly associated with 3 -year overall survival rates (Table II).

Multivariate analysis demonstrated that tumor location (HR, 2.818; 95\% CI, 1.173-6.770; $\mathrm{P}=0.021$ ), histopathological grading $(\mathrm{HR}, 1.907 ; 95 \% \mathrm{CI}, 1.07-3.389 ; \mathrm{P}=0.028)$, TNM stage (HR, 4.801; 95\% CI, 1.912-12.053; $\mathrm{P}=0.001)$ and ZNF365 expression (HR, 0.386; 95\% CI, 0.152-0.980; $\mathrm{P}=0.045)$ were all statistically significant prognostic factors in CRC (Table III). Collectively, these results indicate that ZNF365 may be a valuable prognostic factor in CRC.

Correlation between ZNF365 expression and p53 expression in CRC patients. p53 plays a significant role in the development of tumors (23). The GeneMANIA database was used to assess the association between ZNF365 and p53 expression levels. The results demonstrated that ZNF365 can interact with p53 via RPRM and MAP4 (Fig. 3).

IHC analysis was performed to confirm the correlation between ZNF365 protein expression and total p53 and P-p53 (Ser15) protein expression in 120 cases of CRC. No significant correlation was observed between ZNF365 expression and total p53 expression, while ZNF365 expression was positively correlated with P-p53 (Ser15) protein expression, with a correlation coefficient of $0.189(\mathrm{P}=0.038$; Table IV). Representative IHC staining images of ZNF365 and P-p53 (Ser15) in CRC tissues are presented in Fig. 4.

ZNF365 is downregulated bymethylation inmost CRC cell lines and tissues. In order to determine the molecular mechanism by which ZNF365 expression is decreased in CRC, the cBioPortal database was used to assess the association between ZNF365 expression and DNA methylation. The results demonstrated a statistically significant negative correlation between ZNF365
Table II. Univariate survival analysis of prognostic factors in colorectal cancer.

\begin{tabular}{|c|c|c|c|}
\hline Characteristics & HR & $95 \% \mathrm{CI}$ & P-value \\
\hline Sex & & & 0.419 \\
\hline Male vs. Female & 1.468 & $0.579-3.723$ & \\
\hline Age, years & & & 0.561 \\
\hline$\geq 63$ vs. $<63$ & 1.277 & $0.560-2.912$ & \\
\hline Tumor location & & & 0.067 \\
\hline Rectum vs. Colon & 2.148 & $0.948-4.871$ & \\
\hline Histopathological grading & & & $0.011^{\mathrm{a}}$ \\
\hline Well vs. Moderate vs. Poor & 1.914 & $1.163-3.15$ & \\
\hline Depth of invasion & & & $0.044^{\mathrm{a}}$ \\
\hline $\mathrm{T} 1+\mathrm{T} 2$ vs. $\mathrm{T} 3+\mathrm{T} 4$ & 2.629 & $1.025-6.744$ & \\
\hline Lymph node metastasis & & & $<0.001^{\mathrm{b}}$ \\
\hline N0 vs. N1/2 & 3.111 & $1.84-5.258$ & \\
\hline Distant metastasis & & & $<0.001^{\mathrm{b}}$ \\
\hline M0 vs. M1 & 6.326 & $2.792-14.496$ & \\
\hline TNM stage & & & $<0.001^{\mathrm{b}}$ \\
\hline I/II vs. III/IV & 2.863 & $1.723-4.756$ & \\
\hline ZNF365 expression & & & $0.013^{\mathrm{a}}$ \\
\hline Low vs. High & 0.324 & $0.133-0.787$ & \\
\hline
\end{tabular}

${ }^{\mathrm{a}} \mathrm{P}<0.05$; ${ }^{\mathrm{b}} \mathrm{P}<0.001$. HR, hazard ratio; $\mathrm{CI}$, confidence interval; TNM, tumor-node-metastasis.

Table III. Multivariate survival analysis of prognostic factors in colorectal cancer.

\begin{tabular}{lccc}
\hline Characteristic & HR & $95 \%$ CI & P-value \\
\hline $\begin{array}{l}\text { Sex } \\
\quad \text { Male vs. Female }\end{array}$ & 1.149 & $0.424-3.115$ & 0.784 \\
$\begin{array}{l}\text { Age, years } \\
\quad \text { 63 vs. <63 }\end{array}$ & 1.249 & $0.524-2.973$ & \\
$\quad \begin{array}{l}\text { Tumor location } \\
\text { Rectum vs. Colon }\end{array}$ & 2.818 & $1.173-6.770$ & \\
$\begin{array}{l}\text { Histopathological grading } \\
\text { Well vs. Moderate vs. Poor }\end{array}$ & 1.907 & $1.073-3.389$ & \\
$\begin{array}{l}\text { Depth of invasion } \\
\text { T1 vs. T2+T3+T4 }\end{array}$ & 0.582 & $0.193-1.753$ & \\
$\begin{array}{l}\text { TNM stage } \\
\text { I+II vs. III+IV }\end{array}$ & & & $0.021^{\mathrm{a}}$ \\
$\begin{array}{l}\text { ZNF365 expression } \\
\text { Low vs. High }\end{array}$ & 4.801 & $1.912-12.053$ & \\
\hline
\end{tabular}

${ }^{\mathrm{a}} \mathrm{P}<0.05 ;{ }^{\mathrm{b}} \mathrm{P}<0.001$. HR, hazard ratio; $\mathrm{CI}$, confidence interval; TNM, tumor-node-metastasis.

gene expression and DNA methylation (Spearman, -0.32; $\mathrm{P}=1.016 \times 10^{-4}$; Fig. 5). Semi-quantitative RT-PCR analysis was 
Table IV. Correlation between ZNF365 expression and p53 expression in patients with colorectal cancer $(n=120)$.

\begin{tabular}{lccccr}
\hline & & \multicolumn{2}{c}{ ZNF365 expression } & & \\
p53 expression & Number of patients, $\mathrm{n}(\%)$ & Low, $\mathrm{n}(\%)$ & High, $\mathrm{n}(\%)$ & r value & P-value \\
\hline Total p53 & & & & & 0.107 \\
$\quad$ Low & $40(33.333)$ & $21(52.500)$ & $19(47.500)$ & 0.247 \\
High & $80(66.667)$ & $33(41.250)$ & $47(58.750)$ & & 0.038 \\
Phospho-p5 (Ser15) & $52(43.333)$ & $29(55.769)$ & $23(44.231)$ & 0.189 & \\
Low & $68(56.667)$ & $25(36.765)$ & $43(63.235)$ & & \\
High & & & &
\end{tabular}

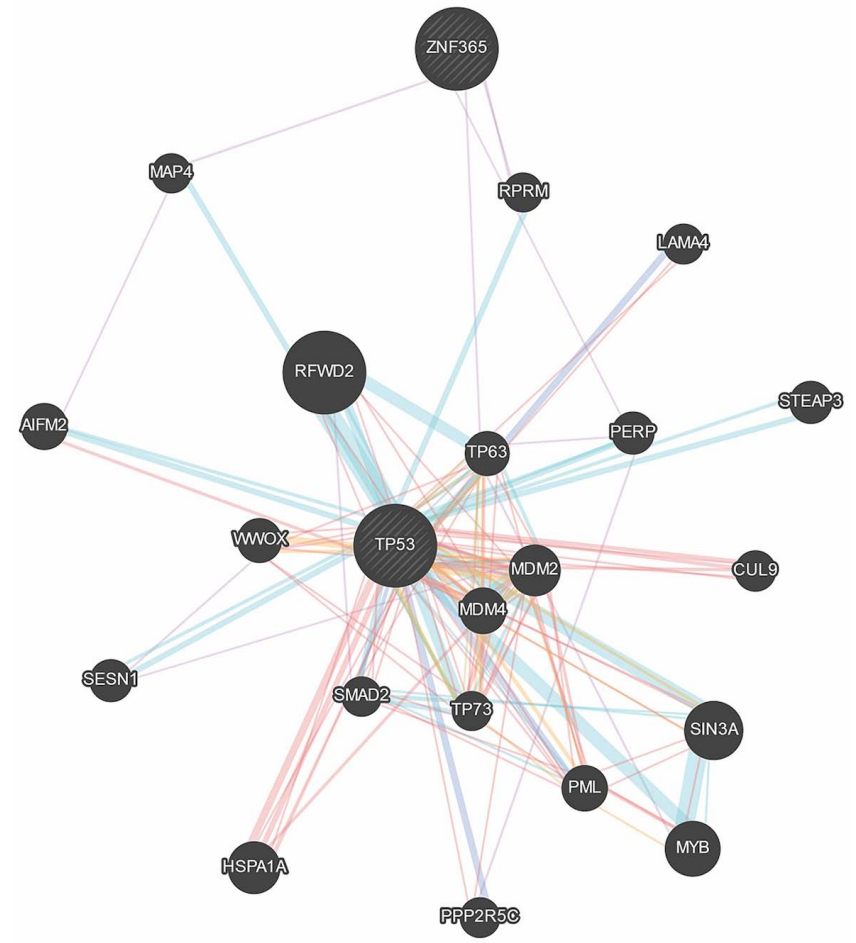

Figure 3. Interaction network between ZNF365 and p53. ZNF365 can interact with p53 through RPRM and MAP4, as predicted by the GeneMANIA database.

subsequently performed to assess ZNF365 expression in CRC cell lines (Colo320, SW620, SW480, LOVO, HCT116, SW48, HCT8, DLD1, HT29 and RKO). The results demonstrated that ZNF365 expression was downregulated or even silenced in most cell lines (Fig. 6A). In addition, MSP primers of ZNF365 were designed to determine its methylation status according to the ZNF365 CpG island (CGI) sequence (Fig. 7A). As expected, the ZNF365 promoter was methylated in the cell lines with decreased ZNF365 expression or ZNF365 silenced (Fig. 6A).

In order to further determine whether promoter methylation directly mediated silencing of ZNF365, its expression was compared before and after treatment in these cell lines, with 5-aza and TSA. The results demonstrated that ZNF365 expression significantly recovered following demethylation treatment in the assessed cell lines, (Fig. 6B). BGS analysis of $49 \mathrm{CpG}$ sites was performed to determine the methylation profiles of
ZNF365 CGI, including those CpG sites analyzed by MSP (Fig. 7). Densely methylated $\mathrm{CpG}$ sites were detected in the cell lines without ZNF365 expression. Both BGS and MSP analyses demonstrated that the ZNF365 CGI was significantly demethylated following TSA and 5-aza treatment, suggesting a direct association between ZNF365 silencing and CGI methylation (Figs. 6B and 7B). Furthermore, MSP analysis was performed to detect ZNF365 methylation in 30 primary colorectal tumors $(\mathrm{T})$ and paired adjacent normal tissues $(\mathrm{N})$. In $63.3 \%$ (19/30) of cases, ZNF365 methylated bands in tumor tissues were stronger than the paired adjacent normal tissues (Fig. 6C), which was confirmed following BGS analysis (Fig. 7B).

\section{Discussion}

ZNF365 serves as a transcription factor, playing key roles in transcriptional activation, protein folding, DNA recognition, lipid binding, RNA packaging and apoptosis regulation (7). The present study aimed to investigate the association between ZNF365 protein expression and the clinicopathological characteristics of patients with CRC. ZNF365, which is predominantly expressed in the cytoplasm and nucleus of CRC tissues (24), was significantly associated with histopathological grading, lymph node metastasis and depth of invasion. However, no significant associations were observed between ZNF365 expression and age, sex, tumor location, distant metastasis or TNM stage. Furthermore, the survival rate of patients with high ZNF365 expression was significantly higher than that of patients with low ZNF365 expression. ZNF365 expression was downregulated in tumor tissues, particularly in poorly differentiated and advanced CRC. The association between ZNF365 expression and CRC from a histological level was also assessed. Multivariate survival analysis demonstrated that ZNF365 protein expression may be used as a prognostic and diagnostic marker for CRC.

Previous studies have reported that genetic polymorphisms of ZNF365 are associated with immune-related diseases in Latin America and Asia $(25,26)$. It has been demonstrated that ZNF365 is associated with atopic dermatitis (AD) in Japanese (26) and overall susceptibility to Crohn's disease in Canadian children (25). Variants of ZNF365 are also associated with susceptibility to breast cancer (16). A total of five SNPs in ADO-ZNF365-EGR2 have been reported to be associated with Vogt-Koyanagi-Harada (VKH) 


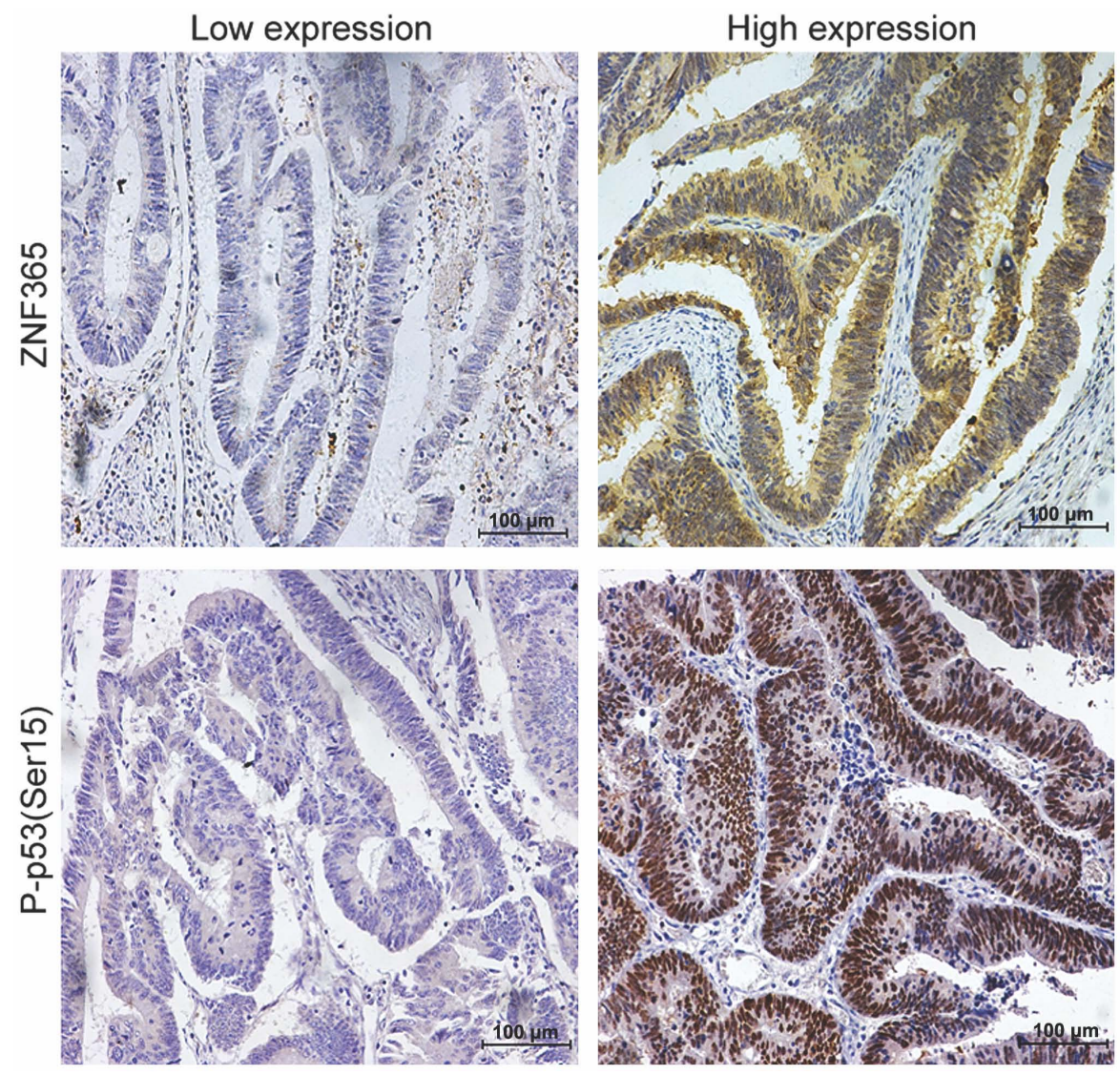

Figure 4. Images of immunohistochemical staining of ZNF365 and P-p53 (Ser15) in CRC tissues. ZNF365 protein expression was positively correlated with P-p53 (Ser15) expression in CRC (magnification, x200). P, phosphorylated; CRC, colorectal cancer.

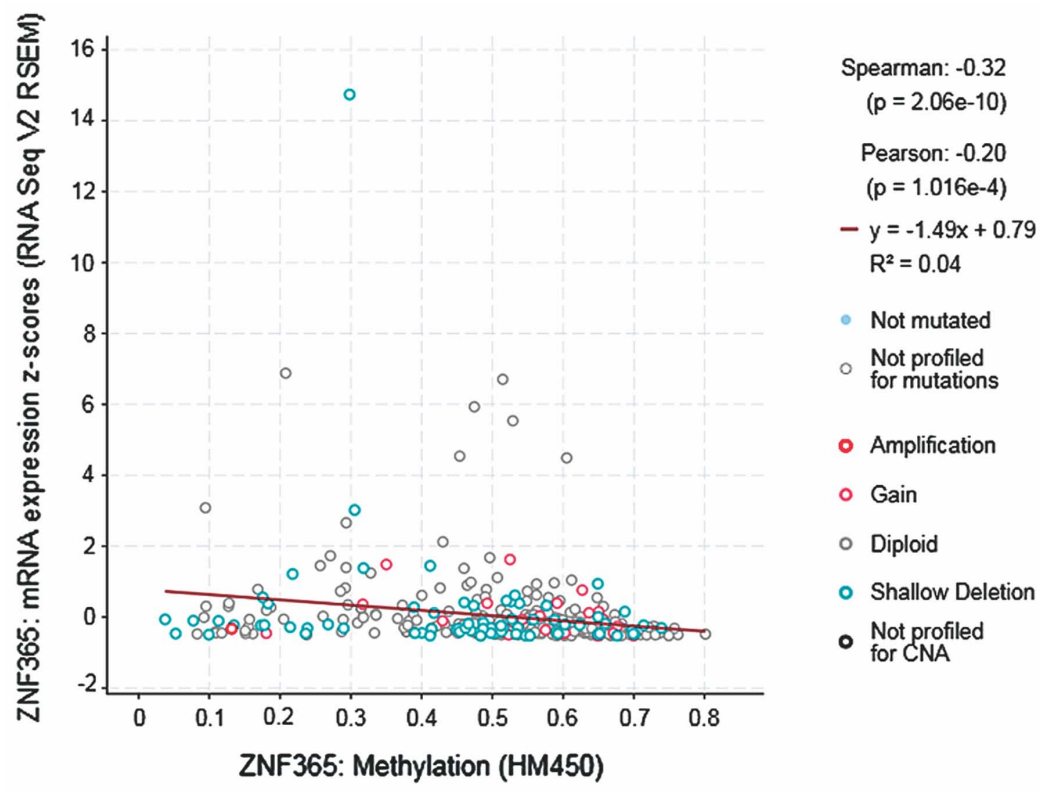

Figure 5. Correlation between ZNF365 mRNA expression and promoter methylation analyzed using the cBioPortal database. There was a significantly negative correlation between ZNF365 gene expression and DNA methylation. Seq, sequence; RSEM, RNA-Seq by Expectation Maximization; CNA, copy number alteration.

syndrome in Thai patients with VKH but not in other Asian patients (15). In addition, Zhang et al (24) demonstrated that ZNF365 acts as a transcriptional target of p53, which is a novel factor caused genomic instability (24). A mechanistic study demonstrated that ZNF365 can suppress expression of a group of common fragile sites including telomeres, and thus, that polymorphisms in the ZNF365 locus are associated with an increased risk of cancer that may result from 


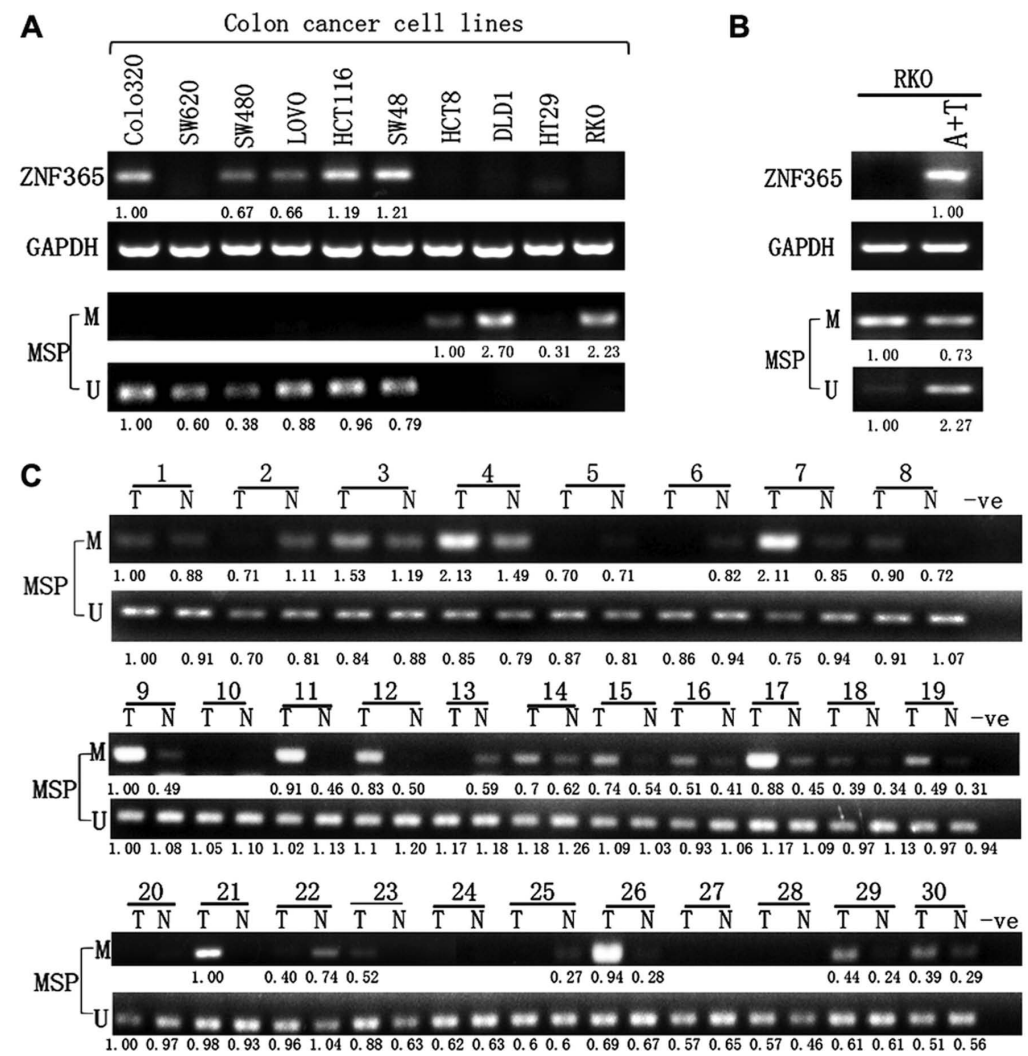

Figure 6. Expression and CGI methylation of ZNF365 in CRC cell lines and primary tumors. (A) ZNF365 expression was downregulated or silenced in several CRC cell lines due to its CGI methylation. (B) Pharmacological demethylation with 5-aza and TSA recovered ZNF365 expression in methylated and silenced cell lines. Typical results are presented. A+T, treatment with 5-aza and TSA. (C) Methylation of ZNF365 in primary T tissues and paired N tissues by MSP. CGI, CpG island; CRC, colorectal cancer; T, tumor; N, non-tumor; M, methylated; U, unmethylated; TSA, trichostatin A; MSP, methylation-specific PCR.

A

getcCCGgct gggtggCGgg ggggtagatg atgttttC' ${ }^{1}$ t tagttgggta

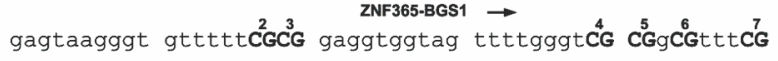

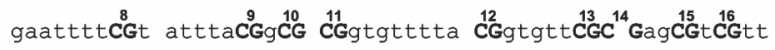

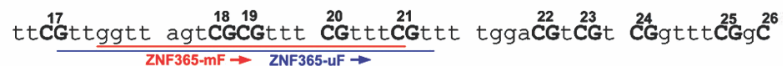

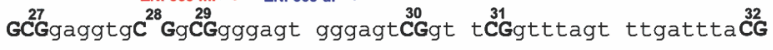

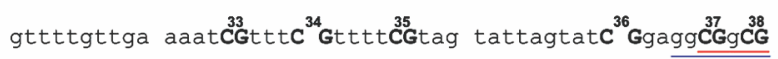

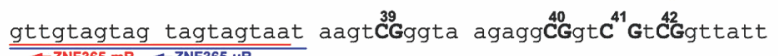

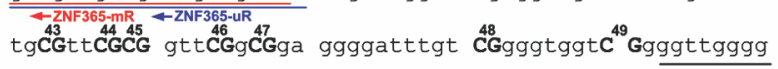
$\underset{\text { agggggtttt }}{\longleftarrow \text { ZNF365-BGS2 }}$
B

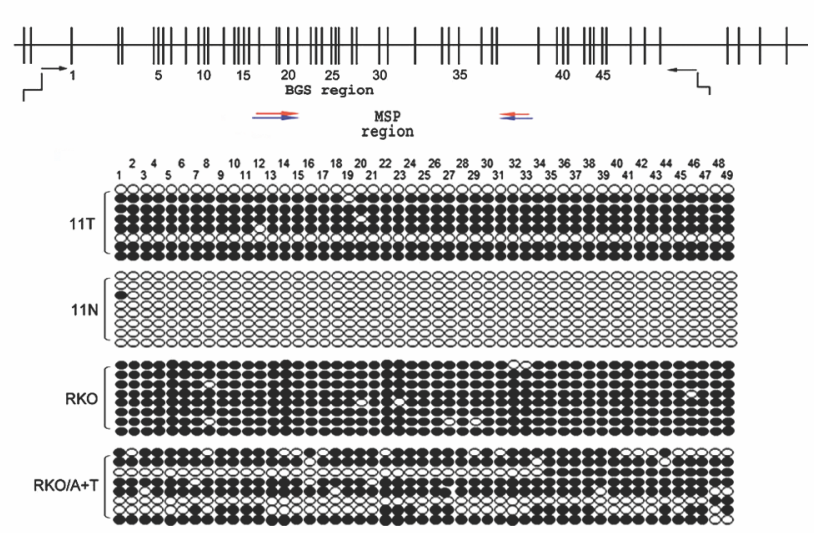

Figure 7. ZNF365 CGI methylation in colorectal cancer cell lines and primary tumors. (A) Sequence of ZNF365 CGI with locations of the 49 CpG sites analyzed and primers used. MSP and BGS regions are also presented. (B) Each vertical line indicates individual CpG sites. Products of cloned BGS-PCR were sequenced and each clone is exhibited as an individual row, representing a single allele of the CGI. Open circles represent unmethylation, while filled circle represent methylation. CGI, CpG island; BGS, bisulfite genomic sequencing.

telomere dysfunction (24). Another study revealed that when induced by DNA double strand break signals, ZNF365 can participate in the homologous recombination repair pathway and maintain genomic integrity during DNA replication by interacting with poly(ADP-ribose) polymerase 1 to tether MRE11 to the DNA end resection site (27). Telomere dysfunction triggers inaccurate DNA repair followed by genomic instability, which is a feature of almost all types of human cancer (28). p53, activated as a transcription factor, plays an important role in preventing tumorigenesis and tumor progression (29). Following DNA damage, p53 protein rapidly accumulates through post-transcriptional mechanisms, which induces growth arrest or apoptosis $(30,31)$. In some cells with defective DNA repair, p53 accumulation is absent or delayed (32). However, mutant p53, detected in $>50 \%$ of cases of solid cancers, loses its ability to specifically bind to sequences of genes that respond to senescence, cell cycle arrest and 
apoptosis, which results in the transformation of this tumor suppressor into an oncogenic factor (33-35). The results of the present study demonstrated that ZNF365 expression did not correlate with total p53 expression; however, it was positively correlated with phospho-p53 (Ser15) expression. Upon DNA damage, p53 is phosphorylated at serine double 15 , which results in decreased interaction of p53 with its negative regulator, the tumor protein MDM2, both in vitro and in vivo (32). This suggests that ZNF365 acts as a suppressor in CRC tumorigenesis and progression by downregulating phospho-p53 (Ser15) expression.

Regarding the expression control mechanisms, the results of the present study demonstrated that ZNF365 expression was downregulated or even silenced in most cell lines. ZNF365 expression was also downregulated in tumor tissues, particularly in advanced cancer and poorly differentiated cancer. Downregulation of ZNF365 expression may be due to its methylation (36), which was detected in $63.3 \%$ of CRC tumors and only $36.7 \%$ of adjacent non-tumor tissues. DNA methylation is a vital component in multilevel gene control in eukaryotes (37). Increasing evidence demonstrates that methylation levels and patterns are deranged in tumor cells $(38,39)$. DNA methylation, together with other epigenetic changes such as RNA editing, affects chromatin structure and thus regulates processes, including allele-specific expression of imprinted genes, $\mathrm{X}$-chromosome inactivation, transcription and inactivation of tumor suppressor genes (40). It was also demonstrated that in the Colo320 and SW620 cell lines, unmethylated alleles coexisted with silencing, suggesting that other expression control mechanisms, such as deletions may also be involved. However, promoter methylation is the main cause of downregulation of ZNF365 (36), and the results of the present study suggest that ZNF365 methylation is a common cancer-specific event in CRC.

The present study is not without limitations. First, as this was a single retrospective study, more research is required to determine the underlying molecular mechanisms between ZNF365 and P-p53. To the best of our knowledge, the present study was the first to investigate the association between ZNF365 expression and CRC. Taken together, the results suggest that downregulation of ZNF365 by methylation may independently predict poor prognosis in patients with CRC, by decreasing P-p53 (Ser15) expression.

\section{Acknowledgements}

Not applicable.

\section{Funding}

The present study was supported by The National Natural Science Foundation of China (grant no. 81672362) and the Test Programs of Science and Technology Commission Foundation of Zhejiang Province (grant no. 2018C37063).

\section{Availability of data and materials}

All data generated or analyzed during this study are included in this published article.

\section{Authors' contributions}

$\mathrm{XH}, \mathrm{CW}, \mathrm{SL}, \mathrm{YK}$ and XF designed the study, analyzed data and approved the version to be published. CW performed the experiments and drafted the manuscript. SL and YK revised it critically for important intellectual content. XF reviewed the manuscript and gave final approval of the version to be published. All authors read and approved the final version of the manuscript.

\section{Ethics approval and consent to participate}

The present study was approved by the Ethics Committee of Sir Run Run Shaw Hospital (Hangzhou, China) and all patients provided written informed consent prior to the study start (approval no. 2019ZNF365-1).

\section{Patient consent for publication}

Patients agreed to the use of their samples in scientific research and provided consent for publication of their information.

\section{Competing interests}

The authors declare that they have no competing interests.

\section{References}

1. Khodavirdipour A, Zarean R and Safaralizadeh R: Evaluation of the anti-cancer effect of Syzygium cumini ethanolic extract on HT-29 colorectal cell line. J Gastrointest Cancer: Jun 6, 2020 (Epub ahead of print).

2. Zoratto F, Rossi L, Verrico M, Papa A, Basso E, Zullo A, Tomao L, Romiti A, Lo Russo G and Tomao S: Focus on genetic and epigenetic events of colorectal cancer pathogenesis: Implications for molecular diagnosis. Tumour Biol 35: 6195-6206, 2014.

3. Palii SS and Robertson KD: Epigenetic control of tumor suppression. Crit Rev Eukaryot Gene Expr 17: 295-316, 2007.

4. Baylin SB and Ohm JE: Epigenetic gene silencing in cancer-a mechanism for early oncogenic pathway addiction? Nat Rev Cancer 6: 107-116, 2006.

5. Krishna SS, Majumdar I and Grishin NV: Structural classification of zinc fingers: Survey and summary. Nucleic Acids Res 31: 532-550, 2003

6. Laity JH, Lee BM and Wright PE: Zinc finger proteins: New insights into structural and functional diversity. Curr Opin Struct Biol 11: 39-46, 2001.

7. Yang L, Hamilton SR, Sood A, Kuwai T, Ellis L, Sanguino A Lopez-Berestein G and Boyd DD: The previously undescribed ZKSCAN3 (ZNF306) is a novel 'driver' of colorectal cancer progression. Cancer Res 68: 4321-4330, 2008.

8. Li J, Wang Y, Fan X, Mo X, Wang Z, Li Y, Yin Z, Deng Y, Luo N, Zhu C, et al: ZNF307, a novel zinc finger gene suppresses p53 and p21 pathway. Biochem Biophys Res Commun 363: 895-900, 2007.

9. Huang C, Jia Y, Yang S, Chen B, Sun H, Shen F and Wang Y: Characterization of ZNF23, a KRAB-containing protein that is downregulated in human cancers and inhibits cell cycle progression. Exp Cell Res 313: 254-263, 2007.

10. Hu R, Peng G, Dai H, Breuer EK, Stemke-Hale K, Li K, Gonzalez-Angulo AM, Mills GB and Lin SY: ZNF668 functions as a tumor suppressor by regulating p53 stability and function in breast cancer. Cancer Res 71: 6524-6534, 2011.

11. Nagase T, Ishikawa K, Suyama M, Kikuno R, Hirosawa M, Miyajima N, Tanaka A, Kotani $\mathrm{H}$, Nomura $\mathrm{N}$ and Ohara $\mathrm{O}$ : Prediction of the coding sequences of unidentified human genes. XII. The complete sequences of 100 new cDNA clones from brain which code for large proteins in vitro. DNA Res 5: 355-364, 1998. 
12. Gianfrancesco F, Esposito T, Ombra MN, Forabosco P, Maninchedda G, Fattorini M, Casula S, Vaccargiu S, Casu G, Cardia F, Deiana I, et al: Identification of a novel gene and a common variant associated with uric acid nephrolithiasis in a Sardinian genetic isolate. Am J Hum Genet 72: 1479-1491, 2003.

13. Medina-Escobedo M, González-Herrera L, Villanueva-Jorge S and Martín-Soberanis G: Metabolic abnormalities and polymorphisms of the vitamin D receptor (VDR) and ZNF365 genes in children with urolithiasis. Urolithiasis 42: 395-400, 2014.

14. Haritunians T, Jones MR, McGovern DP, Shih DQ, Barrett RJ, Derkowski C, Dubinsky MC, Dutridge D, Fleshner PR, Ippoliti A, et al: Variants in ZNF365 isoform D are associated with Crohn's disease. Gut 60: 1060-1067, 2011.

15. Cao S, Chee SP, Yu HG, Sukavatcharin S, Wu L, Kijlstra A, Hou $S$ and Yang P: Investigation of the association of Vogt-Koyanagi-Harada syndrome with IL23R-C1orf141 in Han Chinese Singaporean and ADO-ZNF365-EGR2 in Thai. Br J Ophthalmol 100: 436-442, 2016.

16. Lindström S, Vachon CM, Li J, Varghese J, Thompson D, Warren R, Brown J, Leyland J, Audley T, Wareham NJ, et al: Common variants in ZNF365 are associated with both mammographic density and breast cancer risk. Nat Genet 43: 185-187, 2011.

17. Couch FJ, Gaudet MM, Antoniou AC, Ramus SJ, Kuchenbaecker KB, Soucy P, Beesley J, Chen X, Wang X, Kirchhoff T, et al: Common variants at the 19p13.1 and ZNF365 loci are associated with ER subtypes of breast cancer and ovarian cancer risk in BRCA1 and BRCA2 mutation carriers. Cancer Epidemiol Biomarkers Prev 21: 645-657, 2012.

18. Kleihues P and Sobin LH: World Health Organization classification of tumors. Cancer 88: 2887, 2000.

19. Edge SB and Compton CC: The American Joint Committee on Cancer: The 7th edition of the AJCC cancer staging manual and the future of TNM. Ann Surg Oncol 17: 1471-1474, 2010.

20. Tao Q, Huang H, Geiman TM, Lim CY, Fu L, Qiu GH and Robertson KD: Defective de novo methylation of viral and cellular DNA sequences in ICF syndrome cells. Hum Mol Genet 11: 2091-2102, 2002.

21. Warde-Farley D, Donaldson SL, Comes O, Zuberi K, Badrawi R, Chao P, Franz M, Grouios C, Kazi F, Lopes CT, et al: The GeneMANIA prediction server: Biological network integration for gene prioritization and predicting gene function. Nucleic Acids Res 38 (Web Server Issue): W214-W220, 2010.

22. Ying J, Li H, Seng TJ, Langford C, Srivastava G, Tsao SW, Putti T, Murray P, Chan AT and Tao Q: Functional epigenetics identifies a protocadherin $\mathrm{PCDH} 10$ as a candidate tumor suppressor for nasopharyngeal, esophageal and multiple other carcinomas with frequent methylation. Oncogene 25: 1070-1080, 2006.

23. Naccarati A, Polakova V, Pardini B, Vodickova L, Hemminki K, Kumar R and Vodicka P: Mutations and polymorphisms in TP53 gene-an overview on the role in colorectal cancer. Mutagenesis 27: 211-218, 2012.

24. Zhang Y, Shin SJ, Liu D, Ivanova E, Foerster F, Ying H, Zheng H, Xiao Y, Chen Z, Protopopov A, et al: ZNF365 promotes stability of fragile sites and telomeres. Cancer Discov 3: 798-811, 2013.
25. Amre DK, Mack DR, Morgan K, Israel D, Deslandres C, Seidman EG, Lambrette P, Costea I, Krupoves A, Fegury H, et al: Susceptibility loci reported in genome-wide association studies are associated with Crohn's disease in Canadian children. Aliment Pharmacol Ther 31: 1186-1191, 2010.

26. Hirota T, Takahashi A, Kubo M, Tsunoda T, Tomita K, Sakashita M, Yamada T, Fujieda S, Tanaka S, Doi S, et al: Genome-wide association study identifies eight new susceptibility loci for atopic dermatitis in the Japanese population. Nat Genet 44: 1222-1226, 2012

27. Zhang Y, Park E, Kim CS and Paik JH: ZNF365 promotes stalled replication forks recovery to maintain genome stability. Cell Cycle 12: 2817-2828, 2013.

28. Negrini S, Gorgoulis VG and Halazonetis TD: Genomic instability-an evolving hallmark of cancer. Nat Rev Mol Cell Biol 11: 220-228, 2010

29. Li H, Zhang J, Tong JHM, Chan AWH, Yu J, Kang W and To KF: Targeting the oncogenic p53 mutants in colorectal cancer and other solid tumors. Int J Mol Sci 20: 5999, 2019.

30. Lane DP: Cancer. p53, guardian of the genome. Nature 358: 15-16, 1992.

31. Harris SL and Levine AJ: The p53 pathway: Positive and negative feedback loops. Oncogene 24: 2899-2908, 2005.

32. Shieh SY, Ikeda M, Taya Y and Prives C: DNA damage-induced phosphorylation of p53 alleviates inhibition by MDM2. Cell 91: 325-334, 1997.

33. Soussi T and Beroud C: Assessing TP53 status in human tumours to evaluate clinical outcome. Nat Rev Cancer 1: 233-240, 2001.

34. Sigal A and Rotter V: Oncogenic mutations of the p53 tumor suppressor: The demons of the guardian of the genome. Cancer Res 60: 6788-6793, 2000.

35. Liu YY, Patwardhan GA, Bhinge K, Gupta V, Gu X and Jazwinski SM: Suppression of glucosylceramide synthase restores p53-dependent apoptosis in mutant p53 cancer cells. Cancer Res 71: 2276-2285, 2011.

36. Vedeld HM, Andresen K, Eilertsen IA, Nesbakken A, Seruca R, Gladhaug IP, Thiis-Evensen E, Rognum TO, Boberg KM and Lind GE: The novel colorectal cancer biomarkers CDO1, ZSCAN18 and ZNF331 are frequently methylated across gastrointestinal cancers. Int J Cancer 136: 844-853, 2015.

37. Jones PA: DNA methylation and cancer. Cancer Res 46: 461-466, 1986.

38. Riggs AD and Jones PA: 5-methylcytosine, gene regulation, and cancer. Adv Cancer Res 40: 1-30, 1983.

39. Baylin SB: DNA methylation and gene silencing in cancer. Nat Clin Pract Oncol 2 (Suppl 1): S4-S11, 2005.

40. Moore LD, Le T and Fan G: DNA methylation and its basic function. Neuropsychopharmacology 38: 23-38, 2013.

This work is licensed under a Creative Commons Attribution-NonCommercial-NoDerivatives 4.0 International (CC BY-NC-ND 4.0) License. 